\title{
Safety and feasibility of NeuroFlo use in eight- to 24-hour ischemic stroke patients
}

\author{
M. D. Hammer ${ }^{1,{ }^{*}}$, L. Schwamm ${ }^{2}$, S. Starkman ${ }^{3}$, P. D. Schellinger ${ }^{4}$, T. Jovin ${ }^{1}$, R. Nogueira ${ }^{2}$,

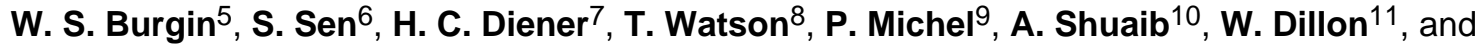 \\ D. S. Liebeskind ${ }^{3}$
}

${ }^{1}$ Department of Neurology, University of Pittsburgh Medical Center, Pittsburgh, PA, USA ${ }^{2}$ Department of Neurology, Massachusetts General Hospital, Boston, MA, USA ${ }^{3}$ Department of Neurology, UCLA, Los Angeles, CA, USA ${ }^{4}$ Department of Neurology, UniversitätsklinikumErlangen, Erlangen, Germany ${ }^{5}$ Department of Neurology, University of Rochester, Rochester, NY, USA ${ }^{6}$ Department of Neurology, University of NC, Chapel Hill, NC, USA ${ }^{7}$ Department of Neurology, Universitätsklinikum Duisburg-Essen, Essen, Germany ${ }^{8}$ Department of Neurology, University of Calgary, Calgary, $\mathrm{AB}$, Canada ${ }^{9} \mathrm{CHUV}$ Lausanne, Lausanne, Switzerland ${ }^{10}$ Stroke Program, University of Alberta, Edmonton, AB, Canada ${ }^{11}$ Department of Radiology, UCSF, San Francisco, CA, USA

\section{Abstract}

Background-Acute treatment of ischemic stroke patients presenting more than eight-hours after symptom onset remains limited and largely unproven. Partial aortic occlusion using the NeuroFlo catheter can augment cerebral perfusion in animals. We investigated the safety and feasibility of employing this novel catheter to treat ischemic stroke patients eight-hours to $24 \mathrm{~h}$ following symptom onset.

Methods-A multicenter, single-arm trial enrolled ischemic stroke patients at nine international academic medical centers. Eligibility included age 18-85 years old, National Institutes of Health stroke scale (NIHSS) score between four and 20, within eight-hours to $24 \mathrm{~h}$ after symptom onset, and perfusion-diffusion mismatch confirmed by magnetic resonance imaging. The primary outcome was all adverse events occurring from baseline to 30 days posttreatment. Secondary outcomes included stroke severity on neurological indices through 90 days. This study is registered with ClinicalTrials.gov, number NCT00436592.

Results-A total of 26 patients were enrolled. Of these, 25 received treatment (one excluded due to aortic morphology); five (20\%) died. Favorable neurological outcome at 90 days (modified Rankin score $0-2$ vs. 3-6) was associated with lower baseline NIHSS $(P<0 \cdot 001)$ and with longer duration from symptom discovery to treatment. There were no symptomatic intracranial

(C) 2012 The Authors. International Journal of Stroke @ 2012 World Stroke Organization

*Correspondence: Maxim Hammer, The Stroke Institute/University of Pittsburgh Medical Center, 200 Lothrop St, PUH-C-419, Pittsburgh, PA 15213, USA. hammermd@upmc.edu.

Conflict of interest: All of the authors are members of the steering committee for the Flo24 clinical trial, which is sponsored by CoAxia, and as such, have received minor payments from CoAxia for consultative work. In addition, the following authors have received major payments from CoAxia for consultative work: Ashfaq Schwaib and Peter Schellinger. 
hemorrhages or parenchymal hematomas. Asymptomatic intracranial hemorrhage was visible on computed tomography in $32 \%$ and only on microbleed in another $20 \%$.

Conclusions-Partial aortic occlusion using the NeuroFlo catheter, a novel collateral therapeutic strategy, appears safe and feasible in stroke patients eight-hours to $24 \mathrm{~h}$ after symptom onset.

\section{Keywords}

acute stroke therapy; cerebral blood flow; cerebral infarction; clinical trial; intervention; stroke

\section{Introduction}

Ischemic stroke remains a devastating clinical event, and few therapeutic strategies exist for its treatment. Thrombolysis with tissue plasminogen activator (tPA) in a time window of three-hours (1), and more recently, up to $4.5 \mathrm{~h}$ (2), has been demonstrated to improve neurological outcome. Thrombectomy, which has not yet definitively proven beneficial in randomized trials, has been increasingly used in an attempt to restore blood flow to the ischemic tissues (3). However, $<5 \%$ of patients with ischemic stroke undergo thrombolysis (4), and $<1 \%$ receive thrombectomy. Alternative therapies are urgently needed, particularly for late-presenting patients and those with contraindications for thrombolysis. Vast efforts have been devoted to arterial revascularization, yet few approaches have capitalized on endogenous collateral circulation as a therapeutic approach $(5,6)$.

Therapeutic titration of the blood flow in the descending aorta to enhance cerebral perfusion has been tested (7). In animals, complete aortic occlusion increases blood volume, particularly in the brain $(8,9)$, reduces infarct size $(10)$, and increases cerebral blood flow (10-12). These and other blood flow manipulation studies led to the development of the NeuroFlo catheter (Fig. 1; CoAxia, Inc., Maple Grove, MN), which increases cerebral perfusion via partial aortic occlusion of the abdominal aorta.

In a study of NeuroFlo use in 24 patients with symptomatic vasospasm, Lylyk et al. demonstrated persistently increased blood flow velocities in the middle cerebral artery (MCA) by transcranial Doppler, with neurological improvement in 83\% of cases (13). In 17 ischemic stroke patients, a one-hour treatment with the NeuroFlo catheter resulted in increases in blood flow velocity in $75 \%$ of the patients, and intraprocedural neurological improvement was noted in $67 \%$ of cases (7).

The ongoing randomized Safety and Efficacy of NeuroFlo Technology in Ischemic Stroke (SENTIS) trial is designed to demonstrate efficacy of NeuroFlo treatment in improving neurological outcome in ischemic stroke patients who can undergo treatment within $14 \mathrm{~h}$ of symptom onset. We were interested to know if late-presenting patients, i.e. those presenting between eight-hours to $24 \mathrm{~h}$, could safely undergo NeuroFlo treatment without an increased risk of intracranial hemorrhage (ICH) or other adverse events (AEs). The Flo 24 study design for late-presenting patients is congruent with many of the recommendations of the Stroke Treatment Academic Industry Roundtable (STAIR) consortium for extended window acute stroke therapy trials (14). 


\section{Materials and methods}

\section{Patient selection}

Patients were eligible for enrollment if they were 18-85 years and presented within eighthours to $24 \mathrm{~h}$ of the last time known normal with acute cerebral ischemia (defined as new focal neurological deficit of presumed vascular origin), with NIHSS 4-20 (inclusive), visual evidence of mismatch $\geq 20 \%$ based on locally constructed perfusion maps, and written informed consent. Exclusion criteria were prior condition resulting in premorbid modified Rankin score (mRS) of $\geq 2$, acute hypodensity of more than one-third of the MCA territory, use of any thrombolytic or thrombectomy therapy, known cerebral or aortic aneurysm, current intracranial bleeding, blood pressure greater than $200 \mathrm{mmHg}$ systolic or $120 \mathrm{mmHg}$ diastolic, or $\geq 5$ microbleeds (MRs) seen only on magnetic resonance (MR) imaging. Laboratory exclusions included a creatinine $>1.5 \times$ local lab normal, international normalized ratio $(\mathrm{INR})>1 \cdot 7$, and platelet count $<100000$. Cardiac exclusions included known ejection fraction $<30 \%$ or current congestive heart failure, known aortic regurgitation $23+$, and current or recent (three-months)myocardial infarction or class III/IV angina. Baseline evaluation required MR angiography and multiparametric MR imaging [diffusion weighted imaging (DWI), perfusion weighted imaging (PWI), and gradient echo (GRE) required; fluid-attenuated inversion recovery (FLAIR) optional]; CT angiography with perfusion imaging was substituted in a few select cases.

Consenting patients meeting all selection criteria underwent an aortogramto determine aortic anatomy. Patients with aortic diameters $>24 \mathrm{~mm}$ or $<12 \mathrm{~mm}$ within six-centimeters above and below the renal ostia, evidence of aortic aneurysm, high-grade iliac stenosis, and/or vascular tortuosity were excluded from further participation (except AE monitoring through hospital discharge).

\section{Treatment technique}

Patients who met all selection criteria and continued to have an NIHSS of 4-20 on repeat examination underwent Neuro-Flo treatment. The dual balloon catheter was placed in the abdominal aorta via standard 9F femoral introduction. Cerebral flow diversion was accomplished by approximately $70-90 \%$ occlusions immediately above and below the renal arteries by independently inflatable balloons. During sequential inflation (infrarenal first), suprarenal pressure was monitored via the catheter central lumen and infrarenal pressure via the introducer side port. The balloons were inflated until each created a detectable $(>5$ $\mathrm{mmHg}$ ) pressure drop with a total incremental pressure drop of at least $10-15 \mathrm{mmHg}$ across both balloons. The balloons were left inflated for 45 mins, slowly deflated, and the catheter removed. Use of heparin was left to the discretion of the interventionalist.

\section{Imaging and clinical end points}

Patients underwent follow-up NIHSS and multimodal MR (DWI and PWI required, FLAIR and GRE optional) within four-hours of treatment, as well as observation for AEs, including groin hematoma or limb ischemia. NIHSS and checks for AEs were documented at $24 \mathrm{~h}$ and at discharge or four-days, which ever came first. Noncontrast CT or MR was performed at $24 \mathrm{~h}$ and as clinically indicated for assessment of bleeding and at four-days/discharge for 
determination of final infarct volume. Neurological indices (NIHSS, Barthel Index, Glasgow Outcome Score, and mRS) were performed at 30 and 90 days. Medical management of the patient throughout the study period was in accordance with the Stroke Council Guidelines.

All imaging scans obtained throughout the index hospitalization were evaluated by a core imaging laboratory (W. Dillon, University of California San Francisco). Baseline scans were evaluated to determine the location and size of the stroke, to uniformly quantify the $\geq 20 \%$ mismatch requirement for study entry, and to determine vessel stenosis/occlusion. Patients were considered to have large vessel occlusion (LVO) if they had thrombolysis in myocardial infarction 0 or 1 flow in the M1 segment or an M2 division of the MCA (15). Patients were considered to have clinically relevant internal carotid artery (ICA) stenosis or occlusion if vessel was $\geq 95 \%$ stenosed or occluded.

All baseline and follow-up scans were also evaluated by the core lab for evidence of ICH, which was further classified as 'other', i.e., ICH not associated with hemorrhagic transformation (HT) of the core infarct, or as one of the four categories of HT used for the European Cooperative Acute Stroke Study (ECASS) I and ECASS II trials (16).

\section{Statistical analysis}

Categorical variables were tabulated as $n(\%)$; continuous variables were tabulated as means with standard deviations, and medians with first and third quartiles. Univariate associations between baseline characteristics and 90-day outcome (where 'better' outcome was defined as mRS 0-2, and 'worse' outcome was defined as mRS 3-6) were evaluated using the Mann-Whitney $U$-test for continuous variables and the Fisher's exact test for categorical variables.

\section{Results}

\section{Patient population}

Altogether, 26 patients were enrolled between May 2007 and September 2008; one was excluded due to aortic anatomy and is not included in these results. No patients were lost to follow-up. Demographic and baseline characteristics of the 25 treated patients are presented in Table 1. Median age was 60.7 years (mean $63.4 \pm 13 \cdot 2$ ), NIHSS was 12 (mean $12.8 \pm$ 4.9), and time from last known normal to treatment was $14 \cdot 2 \mathrm{~h}$ (mean $15 \cdot 7 \pm 4 \cdot 2$ ).We calculated the time from symptom recognition or discovery (TFSD) in addition to determining the time the patient was last known normal (TLKN).As expected, the TFSD (median 11.1, mean 12.4 $\pm 5 \cdot 2$ ) was shorter than TLKN.

Core laboratory examination of baseline imaging confirmed that all patients had $\geq 20 \%$ mismatch (minimum was 38\%), with a PWI volume $>10 \mathrm{ml}$ at baseline (minimum was 54 $\mathrm{ml})$. LVO (17 M1 and four M2 occlusions) was present in 21 of the 25 treated patients (84\%).Compared with the overall cohort, patients with LVO were slightly older (median 62.6 years, mean $63.0 \pm 13 \cdot 1$ ), with similar NIHSS (median 12, mean 13.2 \pm 4.9 ) and TFSD (median $10 \cdot 5 \mathrm{~h}$, mean $12 \cdot 2 \pm 4 \cdot 8$ ). 
Notably, 15 patients (60\%) had ICA occlusion $295 \%$, and 13 (52\%) had both LVO and ICA occlusion. When compared with the overall cohort, patients with ICA occlusion were older (median 64.8 years, mean $65.4 \pm 13.9$ ) and had higher NIHSS (median 16, mean 14.6 \pm 4.7 ) but similar TFSD (median $10.5 \mathrm{~h}$, mean $11.9 \pm 5.6$ ). Table 2 provides each patient's LVO, ICA, and combined LVO plus ICA occlusion status, along with 90-day mRS.

To show potential baseline correlates with 90-day outcomes, Table 1 includes demographics and baseline characteristics by 90 -day outcome status (mRS 0-2 vs. $>2$ ). Worse 90-day outcomes were significantly associated with higher NIHSS, higher infarct volume, and the presence of ICA or ICA + LVO occlusion. Patients with better outcomes had a significantly longer TFSD than patients with worse outcomes, but TLKN was not different. Finally, there was a trend toward better outcomes in patients with prior TIA.

As expected, favorable 90-day outcomes (mRS 0-2) were also associated with favorable 90day Barthel, Glasgow, and NIHSS.

\section{Adverse outcomes through 90 days}

Among the 25 treated patients in the trial, 10 had one or more serious AEs (SAEs). SAEs were events that were fatal, life-threatening, required or prolonged hospitalization, resulted in neurological deterioration considered clinically relevant, or required intervention to prevent permanent impairment as adjudicated by the Data and Safety Monitoring Board (DSMB). There were five patient deaths (20\%); in all cases, death was adjudicated by the DSMB to be related to index stroke progression or to systemic complications associated with index stroke rather than device-related.

Neurological AEs (AEs) included cerebral edema (2), seizure (1), new ischemic stroke (1), and stroke progression (8). All were considered to be nonserious except for the cases of stroke progression where the progression was adjudicated to be serious in six patients. Vascular complications were noted in five patients: one pseudoaneurysm five-days after NeuroFlo treatment in a patient with one prior and one subsequent groin stick (all done in same location), one deep venous thrombosis (DVT), one hematoma, one bleeding at the access site, and one left thigh abscess requiring only oral antibiotics in a patient with history of frequent abscesses. The pseudoaneurysm and DVT were adjudicated as serious.

SAEs beyond those mentioned earlier include one respiratory failure, one infectious pneumonia, two events in one patient of delirium/confusion, one ICA stenosis with endarterectomy, one gastrointestinal bleed, one anemia, and one sepsis. Of note, no patient experienced a serious (or symptomatic) ICH; core lab review of any ICH seen on all patients' imaging is summarized in the following section.

The DSMB also adjudicated device and procedure relatedness for all events. To be adjudicated as device-related, the event was considered likely related to the NeuroFlo device itself; to be adjudicated as procedure-related, the event was considered likely related to any interventional procedure with a 9F sheath. No deaths were adjudicated as device or procedure-related. There were no AEs or SAEs that were adjudicated as device-related. No SAEs were considered to be procedure-related; three AEs were considered to be procedure- 
related (one hematoma, one bleeding at the access site, and one left thigh abscess). Of note, there were no neurologic, cardiac, renal, or pulmonary events considered to be procedure or device-related.

\section{$\mathrm{ICH}$}

ICH data are presented by patient in Table 3 and are summarized in Table 4. None of the ICHs $(n=13)$ were associated with an increase of NIHSS of four or more. No patients had parenchymal hematoma ( $\mathrm{PH} 2)$ grade bleeding. Twelve bleeds were in the index infarction and one was in a new infarction. Of these, 13 patients with new ICH, nine (69\%) had HI1 bleeding, three (23\%) had hemorrhagic infarction (HI2) with one of these in a new infarct at seven-days, and one (8\%) had HI1 within $24 \mathrm{~h}$ that progressed to PH1 on day 2. In the nine patients with HI1 bleeding, five bleeds (56\%) were detected only on MR (no CT performed). In the subset of 12 patients with good 90-day outcomes, four (33.3\%) had a new asymptomatic ICH (three HI1 and one HI2). Two of these ICH were detected only on MR (CT not performed). In the subset of 13 patients with poor 90-day outcomes, nine (69\%) had a new ICH (six HI1, two HI2, and one PH1). Three of the HI1 ICHs in patients with poor outcome were seen only on MR (CT not performed).

\section{Discussion}

This study successfully demonstrates the feasibility and safety of applying partial aortic occlusion using the NeuroFlo catheter in acute ischemic stroke patients who present very late.

Not surprisingly, 90-day clinical outcome was highly correlated with baseline NIHSS score. However, an unexpected correlation was found: patients with better outcomes had a longer average TFSD than those with worse outcomes. Also, there was a trend toward better outcome in patients with prior TIA.

The primary outcome of the trial, safety, was met. No AEs or SAEs were attributed to NeuroFlo treatment. Furthermore, no patients had PH2 or symptomatic ICH (sICH). For comparison, in Prourokinase in Acute Thromboembolic Stroke (PROACT) II, the overall sICH rate was $10 \%$, and among the controls (similar to Flo 24 patients), it was $2 \%$. Within the Diffusion and Perfusion Imaging Evaluation for Understanding Stroke Evolution (DEFUSE) study, the overall sICH rate was $9.5 \%$, and even the subset of patients with mismatch but without successful reperfusion (thus similar to Flo 24 patients) had an sICH rate of 6.3\%. It should be noted, however, that the DEFUSE investigators included any change in NIHSS score as part of their definition for sICH (17).

New asymptomatic bleeding occurred in 13 patients (52\%) in our study, and most of these were HI1. One reason may be that nearly $40 \%$ of the bleeds were detected only on the gradient echo (GRE) sequence on MR, and GRE may be particularly sensitive to HI1. Second, in $56 \%$ of HI1 cases, the bleed was only detected late (beyond two-days). Since HI1 is a marker of successful reperfusion, perhaps the delayed HI1 indicated a delayed reperfusion, possibly influenced by the NeuroFlo treatment. Third, HI may simply be a marker of large strokes. 
This small pilot trial was not designed to test efficacy of NeuroFlo treatment and had no control arm. Considering that our patients had a high rate of LVO, with 21 (84\%) having MCA (M1 or M2) occlusion, and that patients were treated long after stroke onset, poor outcomes may have been predicted. In fact, $40 \%$ of patients at 30 days and $48 \%$ at 90 days (all with mismatch) achieved a good outcome, while $24 \%$ of patients at 30 days and $44 \%$ at 90 days achieved NIHSS $0-1$ or a decrease of eight points from baseline.

There may be several explanations for the favorable outcomes after late NeuroFlo treatment. One is that the treatment itself may have stabilized an inherent progression to collateral failure and infarct evolution in the setting of LVO. An alternative explanation hinges on our observation that outcome was inversely related to TFSD. Perhaps later presenting patients have inherently better collateral circulation that acts to 'pre-serve' the mismatch longer, resulting in milder deficits with still-treatable tissue. The association with prior TIA may be consistent with this hypothesis of inherently better collaterals or could conceivably be related to some ischemic preconditioning that may have promoted development of new collaterals. This association could also be due to chance.

This study is limited by its small size and its lack of a control arm. It is difficult in such a single-arm study to find appropriate populations with which to compare AE rates and outcomes, and the validity of such comparisons is uncertain. Having established the feasibility and safety of this technique along with a signal suggesting efficacy, a randomized trial of late flow augmentation is warranted.

\section{Acknowledgments}

The authors (initials in parentheses) thank Songling Liu, MD, UCSF, San Francisco, CA (WD), for outstanding core lab help. They also acknowledge the work of the entire Flo 24 research team from each participating site (number enrolled + additional number consented): University of Pittsburgh, Pittsburgh, PA (9 + 8, M. D. H., T. J.); UCLA, Los Angeles, CA (5 + 1, S. S., D. L.); Universitätsklinikum-Erlangen, Erlangen, Germany ( 3 + 0, P. S.); Massachusetts General Hospital, Boston, MA ( $2+1$, L. S., R. N.); University of Rochester, Rochester, NY ( $2+1$, W. S. B.); Universitätsklinikum Duisburg-Essen, Essen, Germany (2 + 1, H. C. D.); University of NC, Chapel Hill, NC (1 + 3, S. S.); University of Calgary, Calgary, AB, Canada (1 + 0, T. W.); CHUV Lausanne, Lausanne, Switzerland (1 + 1, P. M.); University of Alberta, Edmonton, AB, Canada $(0+6$, A. S.); Universitätsklinikum Mannheim, Germany $(0+2)$; InselSpital Bern, Switzerland $(0+1)$; ZNA Middelheim, Antwerp, Belgium $(0+1)$.

Funding: Funding for this study was provided by CoAxia, Inc., Maple Grove, MN. DSL received grant support from NINDS K23054084.

\section{References}

1. The National Institute of Neurologic Disorders and Stroke rt-PA Stroke Study Group. Tissue plasminogen activator for acute ischemic stroke. N Engl J Med. 1995; 333:1581-1587. [PubMed: 7477192]

2. Hacke W, Kaste M, Bluhmki E, et al. for the ECASS Investigators. Thrombolysis with alteplase 3. to 4.5. hours after acute ischemic stroke. N Engl J Med. 2008; 359:1317-1329. [PubMed: 18815396]

3. Nogueira RG, Yoo AJ, Buonanno FS, Hirsch JA. Endovascular approaches to acute stroke, part 2: a comprehensive review of studies and trials. AJNR Am J Neuroradiol. 2009; 30:859-875. [PubMed: 19386727]

4. Furlan A. IV Tissue Plasminogen Activator for stroke in the community. What we know and don't know 10. years after FDA approval. Stroke. 2006; 37:281. [PubMed: 16397163] 
5. Liebeskind D. Collateral therapeutics for cerebral ischemia. Expert Rev Neurother. 2004; 4:255265. [PubMed: 15853567]

6. Liebeskind D. Collaterals in acute stroke: beyond the clot. Neuroimaging Clin N Am. 2005; 15:553573. [PubMed: 16360589]

7. Liebeskind D. Aortic occlusion for cerebral ischemia: from theory to practice. Curr Cardiol Rep. 2008; 10:31-36. [PubMed: 18416998]

8. Stokland O, Miller MM, Ilebekk A, Kiil F. Mechanism of hemodynamic responses to occlusion of the descending thoracic aorta. Am J Physiol. 1980; 238:H423-H429. [PubMed: 7377312]

9. Saether OD, Juul R, Aadahl P, Strømholm T, Myhre HO. Cerebral haemodynamics during thoracic and throacoabdominal aortic aneurysm repair. Eur J Vasc Endovasc Surg. 1996; 12:81-85. [PubMed: 8696903]

10. Noor R, Wang CX, Todd K, Elliott C, Wahr J, Shuaib A. Partial intraaortic occlusion improves perfusion deficits and infarct size following focal cerebral ischemia. J Neuroimaging. 2009; 20:15. (accepted for publication, available online). [PubMed: 19747237]

11. Simeone FA. Enhancement of cerebral blood flow by intermittent aortic occlusion. Eur Neurol. 1972; 8:142-144. [PubMed: 4626352]

12. Hammer M, Jovin T, Wahr JA, Heiss W-D. Partial occlusion of the descending aorta increases cerebral blood flow in a nonstroke porcine model. Cerebrovasc Dis. 2009; 28:406-410. [PubMed: 19713700]

13. Lylyk P, Vila JF, Miranda C, Ferrario A, Romero R, Cohen J. Partial aortic obstruction improves cerebral perfusion and clinical symptoms in patients with symptomatic vasospasm. Neurol Res. 2005; 27:S129-S135. [PubMed: 16197838]

14. Saver JL, Albers GW, Dunn B, Johnson KC, Fisher M. for the STAIR VI Consortium. Stroke Therapy Academic Industry Roundtable (STAIR) recommendations for extended window acute stroke therapy trials. Stroke. 2009; 40:2594-2600. [PubMed: 19478212]

15. Furlan A, Higashida R, Wechsler L, et al. for the PROACT Investigators. Intra-arterial prourokinase for acute ischemic stroke. The PROACT II study: a randomized controlled trial. JAMA. 1999; 282:2003-2011. [PubMed: 10591382]

16. Berger C, Fiorelli M, Steiner T, et al. Hemorrhagic transformation of ischemic brain tissue: asymptomatic or symptomatic? Stroke. 2001; 32:1330-1335. [PubMed: 11387495]

17. Albers GW, Thijs VN, Wechsler L, et al. for the DEFUSE Investigators. Magnetic resonance imaging profiles predict clinical response to early reperfusion: the Diffusion and Perfusion Imaging Evaluation for Understanding Stroke Evolution (DEFUSE) Study. Ann Neurol. 2006; 60:508-517. [PubMed: 17066483] 


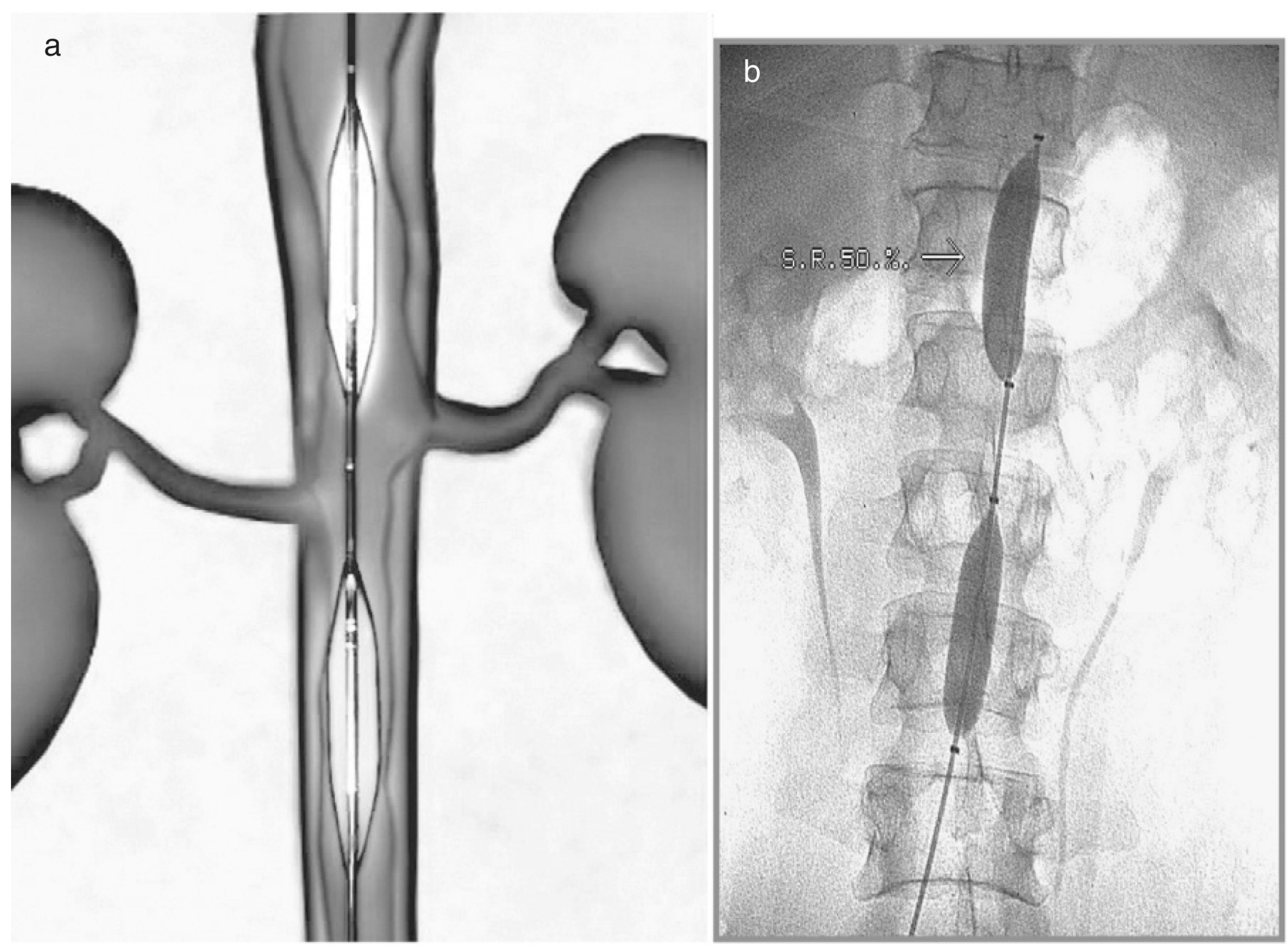

Fig. 1.

(a) Schematic of NeuroFlo; (b) NeuroFlo in the aorta. 


\section{Table 1}

\section{Patient demographics}

\begin{tabular}{|c|c|c|c|c|}
\hline Characteristic & All, $n=25(\%)$ & mRS 0-2, $n=12(\%)$ & $\mathrm{mRS}>2, n=13(\%)$ & $P$-value \\
\hline \multicolumn{5}{|l|}{ Age, years } \\
\hline Median [first quartile, third quartile] & $60 \cdot 7[54 \cdot 1,72 \cdot 0]$ & $61 \cdot 2[54 \cdot 9,69 \cdot 9]$ & $60 \cdot 7[53 \cdot 2,74 \cdot 1]$ & $1 \cdot 000$ \\
\hline Mean \pm standard deviation & $63 \cdot 4 \pm 13 \cdot 2$ & $62 \cdot 7 \pm 10 \cdot 5$ & $64 \cdot 0 \pm 15 \cdot 8$ & \\
\hline \multicolumn{5}{|l|}{ Gender } \\
\hline Male & $11(44 \cdot 0)$ & $6(50 \cdot 0)$ & $5(38 \cdot 5)$ & $0 \cdot 695$ \\
\hline Female & $14(56 \cdot 0)$ & $6(50 \cdot 0)$ & $8(61 \cdot 5)$ & 0.695 \\
\hline \multicolumn{5}{|l|}{ Race } \\
\hline Caucasian & $22(88 \cdot 0)$ & $10(83 \cdot 3)$ & $12(92 \cdot 3)$ & $0 \cdot 593$ \\
\hline African American & $3(12 \cdot 0)$ & $2(16 \cdot 7)$ & $1(7 \cdot 7)$ & \\
\hline \multicolumn{5}{|l|}{ Baseline NIHSS $*$} \\
\hline Median [first quartile, third quartile] & $12 \cdot 0[9 \cdot 0,17 \cdot 0]$ & $9 \cdot 0[7 \cdot 0,11 \cdot 0]$ & $17 \cdot 0[13 \cdot 0,18 \cdot 0]$ & $<0 \cdot 001$ \\
\hline Mean \pm standard deviation & $12 \cdot 8 \pm 4 \cdot 9$ & $9 \cdot 1 \pm 3 \cdot 0$ & $16 \cdot 2 \pm 3 \cdot 8$ & \\
\hline \multicolumn{5}{|c|}{ Time from symptom discovery to treatment, hours } \\
\hline Median [first quartile, third quartile] & $11 \cdot 1[8 \cdot 8,14 \cdot 4]$ & $14 \cdot 3[10 \cdot 7,19 \cdot 9]$ & $9 \cdot 9[8 \cdot 5,11 \cdot 1]$ & $0 \cdot 030$ \\
\hline Mean \pm standard deviation & $12 \cdot 4 \pm 5 \cdot 2$ & $15 \cdot 0 \pm 6 \cdot 0$ & $10 \cdot 0 \pm 3 \cdot 0$ & \\
\hline \multicolumn{5}{|c|}{ Time from last known normal to treatment, hours } \\
\hline Median [first quartile, third quartile] & $14 \cdot 2[13 \cdot 2,18 \cdot 3]$ & $16 \cdot 2[13 \cdot 6,21 \cdot 1]$ & $13 \cdot 5[11 \cdot 1,17 \cdot 8]$ & $0 \cdot 123$ \\
\hline Mean \pm standard deviation & $15 \cdot 7 \pm 4 \cdot 2$ & $17 \cdot 1 \pm 4 \cdot 3$ & $14 \cdot 5 \pm 3 \cdot 8$ & \\
\hline \multicolumn{5}{|c|}{ Baseline infarct volume, $\mathrm{cc}$ (17 NCT and 8 MR scans) } \\
\hline Median [first quartile, third quartile] & $9 \cdot 1[1 \cdot 2,22 \cdot 2]$ & $3 \cdot 3[0 \cdot 0,8 \cdot 8]$ & $21 \cdot 6[9 \cdot 1,61 \cdot 7]$ & $0 \cdot 001$ \\
\hline Mean \pm standard deviation & $26 \cdot 7 \pm 46 \cdot 0$ & $7 \cdot 6 \pm 12 \cdot 1$ & $44 \cdot 3 \pm 58 \cdot 4$ & \\
\hline Large vessel occlusion (LVO) $)^{\dagger}$ & $21(84 \cdot 0)$ & $9(75 \cdot 0)$ & $12(92 \cdot 3)$ & $0 \cdot 322$ \\
\hline ICA occlusion ${ }^{+}$ & $15(60 \cdot 0)$ & $4(33 \cdot 3)$ & $11(84.6 \%$ & $0 \cdot 015$ \\
\hline LVO + ICA occlusion & $13(52 \cdot 0)$ & $3(25)$ & $10(76 \cdot 9)$ & $0 \cdot 017$ \\
\hline History of hypertension & $21(84 \cdot 0)$ & $11(91 \cdot 7)$ & $10(76 \cdot 9)$ & 0.593 \\
\hline History of diabetes & $8(32 \cdot 0)$ & $3(25 \cdot 0)$ & $5(38 \cdot 5)$ & $0 \cdot 673$ \\
\hline Current smoker & $10(40 \cdot 0)$ & $3(25 \cdot 0)$ & $7(53 \cdot 8)$ & $0 \cdot 226$ \\
\hline Prior stroke & $4(16 \cdot 0)$ & $3(25 \cdot 0)$ & $1(7 \cdot 7)$ & $0 \cdot 322$ \\
\hline Prior TIA & $3(12 \cdot 0)$ & $3(25 \cdot 0)$ & $0(0 \cdot 0)$ & 0.096 \\
\hline History of atrial fibrillation & $4(16 \cdot 0)$ & $1(8 \cdot 3)$ & $3(23 \cdot 1)$ & 0.593 \\
\hline History of peripheral vascular disease & $1(4 \cdot 0)$ & $1(8 \cdot 3)$ & $0(0 \cdot 0)$ & $0 \cdot 480$ \\
\hline History of PTCA, CABG, and/or MI & $2(8 \cdot 0)$ & $0(0 \cdot 0)$ & $2(15 \cdot 4)$ & $0 \cdot 480$ \\
\hline
\end{tabular}


Table 2

Baseline vessel occlusion status, by patient, by 90-day outcome

\begin{tabular}{|c|c|c|c|c|}
\hline Patient ID no. & 90-day mRS & LVO per PROACT II definition & ICA occlusion $>95 \%$ & Both LVO and ICA occlusion \\
\hline 18 & 0 & M1 & No & No \\
\hline 3 & 1 & M1 & Yes & Yes \\
\hline 9 & 1 & M1 & Yes & Yes \\
\hline 10 & 1 & M1 & No & No \\
\hline 11 & 1 & no & No & No \\
\hline 21 & 1 & M2 & No & No \\
\hline 22 & 1 & no & No & No \\
\hline 23 & 1 & M2 & Yes & Yes \\
\hline 24 & 1 & M2 & No & No \\
\hline 5 & 2 & M2 & No & No \\
\hline 6 & 2 & no & Yes & No \\
\hline 20 & 2 & M1 & No & No \\
\hline 7 & 3 & M1 & Yes & Yes \\
\hline 16 & 3 & M1 & No & No \\
\hline 25 & 3 & M1 & Yes & Yes \\
\hline 1 & 4 & M1 & Yes & Yes \\
\hline 2 & 4 & M1 & Yes & Yes \\
\hline 13 & 4 & M1 & Yes & Yes \\
\hline 15 & 4 & M1 & Yes & Yes \\
\hline 14 & 5 & M1 & No & No \\
\hline 17 & $6(\mathrm{~d} 3)$ & M1 & Yes & Yes \\
\hline 4 & $6(\mathrm{~d} 10)$ & no & Yes & No \\
\hline 8 & $6(\mathrm{~d} 13)$ & M1 & Yes & Yes \\
\hline 12 & $6(\mathrm{~d} 37)$ & M1 & Yes & Yes \\
\hline 19 & $6(\mathrm{~d} 38)$ & M1 & Yes & Yes \\
\hline
\end{tabular}

mRS scoring: $0=$ no deficits, $6=$ death, with days to death in parentheses.

Large vessel occlusion $(\mathrm{LVO})=$ TIMI 0 or 1 flow in the M1 segment or an M2 division of the middle cerebral artery, per PROACT II definition $(0)$.

ICA occlusion $=\geq 95 \%$ stenosis or occlusion in the ICA ipsilateral to the stroke. 


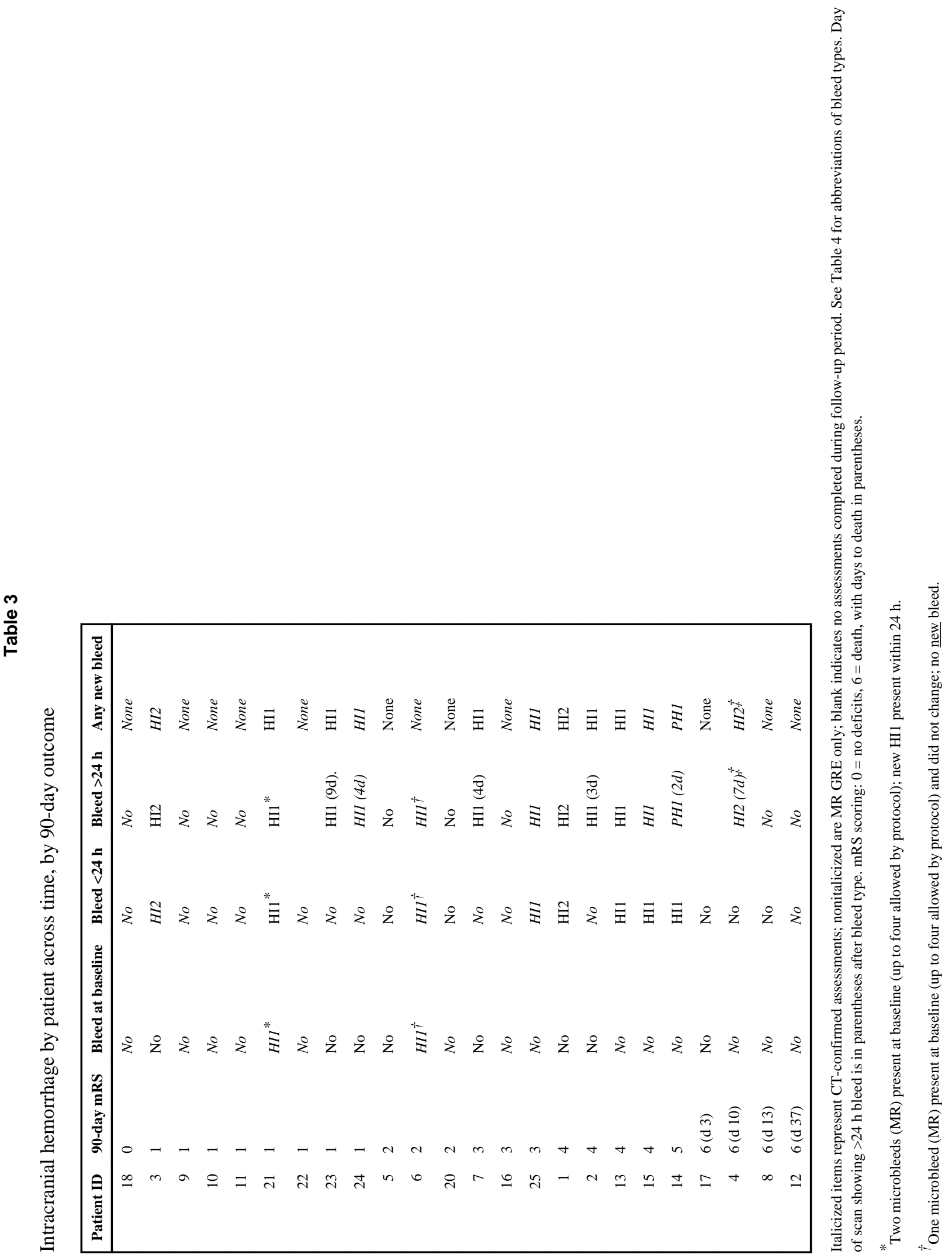




\section{Table 4}

Posttreatment intracranial hemorrhage (ICH) summary

\begin{tabular}{|c|c|c|c|c|}
\hline & $\begin{array}{l}\text { All } \\
n=25(\%)\end{array}$ & $\begin{array}{l}\text { mRS 0-2 } \\
n=12(\%)\end{array}$ & $\begin{array}{l}\text { mRS 3-6 } \\
n=13(\%)\end{array}$ & $P$-value \\
\hline New ICH through $24 \mathrm{~h} n$ & $7 / 25(28)$ & $2 / 12(16 \cdot 7)$ & $5 / 13(38 \cdot 5)$ & $0 \cdot 378$ \\
\hline All new ICH (four-hours through nine-days) $n$ & $13 / 25(52 \cdot 0)$ & $4 / 12(33 \cdot 3)$ & $9 / 13(69 \cdot 2)$ & $0 \cdot 115$ \\
\hline All new ICH confirmed on CT (four-hours through nine-days) $n$ & $8 / 25(32 \cdot 0)$ & $2 / 12(16 \cdot 7)$ & $6 / 13(46 \cdot 2)$ & $0 \cdot 201$ \\
\hline \multirow[t]{4}{*}{ Categories for all new $\mathrm{ICH}$ on any scan } & $9 \mathrm{HI} 1$ & $3 \mathrm{HI} 1$ & $6 \mathrm{HI} 1$ & $0 \cdot 089$ \\
\hline & $3 \mathrm{HI} 2$ & $1 \mathrm{HI} 2$ & $1 \mathrm{HI} 2$ & \\
\hline & $1 \mathrm{PH} 1$ & $0 \mathrm{PH} 1$ & $1 \mathrm{PH} 1$ & \\
\hline & 0 other & 0 other & 0 other & \\
\hline
\end{tabular}

ICH categories: other = ICH not associated with hemorrhagic transformation of the core infarct; HI1 (petechial infarction without space-occupying effect): HI2 (more confluent petechiae); PH1 ( $\$ 30 \%$ of infarcted area with some mild space-occupying effect); $\mathrm{PH} 2$ ( $>30 \%$ of the infarcted area with significant space-occupying effect, or clot remote from infarcted area). 